\title{
Observando las representaciones de medios de comunicación acerca de la educación: el caso de la radio Bío-Bío*
}

\author{
Fernando Farías Olavarría ** \\ Cristian Orellana Fonseca*** \\ Gabriel Pérez Díaz****
}

Recibido: 2016-04-26. Enviado a pares: 2016-05-05

Aprobado por pares: 2016 05-28. Aceptado: 2016-06 10

DOI: 10.22395/angr.v15n29a4

\begin{abstract}
Resumen
El objetivo del estudio es describir la representación social acerca de la educación en un medio de comunicación masivo como es la Radio Bío-Bío. Para ello se construyó un corpus a partir del conjunto de los artículos de prensa publicados durante el año 2013, que fue analizado con procedimientos lexicométricos y de análisis temático de contextos elementales. A través de tales procedimientos, fue posible identificar los principales actores involucrados, sus temáticas impulsadas, y reconocer tres clúster de noticias que marcaron la representación de la educación durante el año 2013: "movilización", "sistema universitario", y "respuesta del sistema político", tendientes a abordar la crisis del sistema educacional.
\end{abstract}

Palabras clave: educación, representación, medios de comunicación cluster discursivos

Este trabajo es parte de los proyectos 125324 3/R de la Dirección de Investigación de la Universidad del Bío-Bío y del Proyecto Sistema Territorial de Educación: Observatorio Prospectivo Educación y Desarrollo Regional 1202 (CHILE).

** Magíster en Investigación Social y Desarrollo, Trabajador Social. Académico del Departamento de Ciencias Sociales, Facultad de Educación y Humanidades, Universidad del Bío-Bío, Chillán, Chile.ffarias@ubiobio. cl.

..* Magíster en Investigación Social y Desarrollo, sociólogo, profesor de Historia y Geografía. Académico del Departamento de Ciencias Sociales, Facultad de Educación y Humanidades, Universidad del Bío-Bío, Chillán, Chile.corellana@ubiobio.cl.

.... Licenciado en Sociología. gabrielperez@udec.cl 


\title{
Observing communication media representation about education: Bío-Bío radio case
}

\begin{abstract}
The object of this study is to describe social representation about education in mass communication media such as Bio-Bio Radio. With this purpose, a corpus from newspaper articles published in the year 2013, was constructed, which was analyzed with lexicometric procedures and thematic elemental contexts. Through these procedures, it was possible to identify the main agents involved, their impulse topics and to recognize three new clusters, which marked the representation of education during the year 2013: "Mobilization," "University system," "Reply of the political system," with the purpose of tackling the crisis of the education system.
\end{abstract}

Key words: education, representation, communication media, discourse clusters. 


\section{Introducción}

Este trabajo se inserta en el marco de las acciones que emprende el Observatorio Prospectivo sobre Educación y Desarrollo de la Universidad del Bío-Bío, para hacer un diagnóstico desde distintos actores que posibilite avanzar en la construcción de los futuros posibles e identificación de estrategias que puedan decantar en propuestas de políticas públicas para contribuir a mejorar la Educación Superior en la región del Biobío.

Desde mediados de la primera década del siglo XXI, la educación chilena ha formado parte de la agenda noticiosa del país. Esta ha dado cuenta de dos grandes hitos, que han tenido como objetivo generar cambios que corrijan las inexorables desigualdades sociales presentes en el sistema educativo: las movilizaciones lideradas por estudiantes secundarios durante el año 2006, y las lideradas por estudiantes universitarios, durante el 2011(Orellana, 2014).

El primero de estos hitos lo constituye en el año 2006 la llamada "revolución pingüina", movimiento de estudiantes secundarios cuyas demandas se encontraban organizadas, por una parte, en una agenda de corto plazo, que contemplaba, entre otras, el uso del pase escolar las 24 horas, mayor número de raciones alimenticias, y la gratuidad de la prueba de selección universitaria (PSU). Por otra parte, sostuvieron una agenda de más largo aliento, que apuntaba a la realización de cambios profundos dentro del sistema educativo implantado por la dictadura cívico-militar a través de la LOCE (1). En definitiva, la demanda consistía en la derogación de la LOCE, con el objetivo de que la administración de la educación retornase al Estado, asegurando así el fortalecimiento de la educación pública como derecho, a través de la gratuidad.

La respuesta del entonces gobierno de Michelle Bachelet (2006-2009) consistió en acudir a la "lógica de los expertos" (Orellana, 2014), convocando a un Consejo Asesor Presidencial que propuso, entre otras medidas, reemplazar la LOCE por la Ley General de Educación (que finalmente fue promulgada en agosto de 2009). Esta nueva ley, a juicio de los estudiantes, no fue suficiente para llevar a cabo los cambios estructurales que el movimiento demandaba.

Con este hecho como antecedente, se constituye así en 2011 el segundo hito, momento en que las movilizaciones estudiantiles resurgen demandando las transformaciones profundas al sistema educacional que -a su juicio- no habían sido abordadas en el período anterior. Esta vez son lideradas por organizaciones de estudiantes universitarios, como la Confederación de Estudiantes de Chile (CONFECH), y, en menor medida, por organizaciones de estudiantes secundarios (2). Estas movilizaciones adquieren un apoyo masivo de la ciudadanía, al punto que empiezan a denominarse "Movimiento Social por la Educación". En el momento donde el conflicto por la educación dominaba comunicacionalmente la agenda informativa, alrededor de agosto de 2011, la aprobación de las demandas del movimiento por parte de la ciudadanía llegó al 76 \%, siendo transversal a todos los segmentos, mientras que la aprobación del gobierno de Sebastián Piñera (2010-2013) respecto del manejo de la situación apenas llegaba al 18 \% (Adimark, 2011). 
Entendemos que la prensa es una institución social que contribuye en la construcción social de sentido (Vásquez, 1999). Desde esta perspectiva, la acción periodística es parte de la construcción y distribución de discursos sociales, la cual puede tender tanto a sedimentar el consenso, como a posicionar el conflicto; esto, de acuerdo con el marco a través del cual se presente una serie de hechos como una realidad, y por la forma en que se aborde su comprensión, análisis y escudriñamiento.

Así es como las cuestiones que giran en torno a los procesos de mediación son dignas de considerar, debido a que los medios de comunicación toman parte en la construcción de realidad, especialmente en los ámbitos relacionados con lo público. Con esto, pueden practicar cierto control social, actuando sobre los usuarios en la cadena interminable de creación de "mundo real" del que se nutren tanto los medios como las sociedades, y cuyo propósito es orientar ciertas conductas para mantener una configuración particular de la realidad social.

En esta línea, consideramos que para estudiar la forma en que los medios de comunicación toman parte de la construcción de realidad, el concepto de representación social resulta pertinente. Las representaciones sociales corresponden a "imágenes que condensan un conjunto de significados, sistemas de referencia que nos permiten interpretar lo que nos sucede, es decir, una manera de interpretar y de pensar nuestra realidad cotidiana" (Jodelet, 1984, p. 472). En consecuencia, pueden ser interpretadas como modelos construidos, que sirven para orientar la acción y la valoración de los sujetos (Martín Serrano, 1977). En este caso, describir las representaciones sociales sobre la educación de un medio masivo de comunicación nos permite acceder a la forma en que se construyó un modelo interpretativo sobre la realidad del conflicto por la educación durante el 2013, y de esta manera, entender cómo podría influir en la forma en que sus lectores orientan sus valoraciones y acciones respecto del mismo tema.

De este modo, la teoría de las representaciones sociales sugerida por Moscovici desde los años sesenta del siglo XX, se ha configurado como uno de los instrumentos fundamentales para la comprensión de la relación entre el discurso y la práctica social. Como se ha indicado anteriormente, las representaciones sociales serían el conjunto de conocimientos construidos sobre hechos y acciones sociales, lo cual hace que los sujetos construyan estilos cognitivos, en el sentido de ordenar cognitiva y afectivamente la realidad (Martinic, 2006).

\section{Material y métodos}

Para llevar a cabo este análisis preliminar de la representación social acerca de la educación en el medio de comunicación, se construyó un corpus de texto a partir de las noticias publicadas en la página web de Radio Bío-Bío (www.biobiochile.cl), durante el período de tiempo correspondiente del 01 de enero al 31 de diciembre de 2013. Las noticias fueron obtenidas a través del mismo buscador del sitio, bajo el término "educación". Esto significó la selección de un total de 430 publicaciones vinculadas a diferentes tópicos educativos. Las noticias fueron clasificadas a partir de su orientación territorial: nacional, regional, 
provincial y comunal. No obstante, el estudio estuvo centrado en las noticias de orientación nacional. Para el logro de los objetivos se recurrió al análisis de contenido lexicométrico y al análisis temático de contextos elementales, por medio del programa T-Lab 8.1.

Se sometió el corpus a un proceso automático de lematización. Esto significa que las palabras (u ocurrencias) que componen el texto fueron agrupadas en términos -o lemasque poseen la misma raíz lexical, y que pertenecen a la misma categoría gramatical, aunque esta norma pueda tener excepciones (como en los verbos irregulares). Por ejemplo, los términos "hablan" y "hablando", remitirían al lema "hablar", pues comparten raíz, y ambos son formas verbales. De este modo, el software entregó un conjunto de lemas sugeridos y excluidos, que fueron revisados por los autores, con la finalidad de asegurar que fueran útiles para responder a los objetivos del estudio en particular. Esto es, se utilizaron los lemas que permitirían identificar de mejor manera actores y temáticas relevantes, a partir de los contextos discursivos en los que estaban insertos, es decir, en los respectivos párrafos de las noticias que formaron nuestro corpus de trabajo.

Para identificar esto, se recurrió al análisis temático de contextos elementales. Al importar el texto, el programa segmenta el corpus en lo que se denomina "contextos elementales". A partir de ellos los lemas adquieren sentido, así como las palabras adquieren sentido en la trama de un texto. Estos contextos pueden definirse como una frase, un fragmento, un párrafo, o un texto breve. En este caso, consideramos el párrafo como contexto elemental, pues nos pareció suficiente para identificar sentido dentro de las noticias.

Luego, se llevó a cabo la representación de los contenidos del corpus mediante clusters temáticos, que no son más que conjuntos que reúnen -en nuestro caso- párrafos de noticias que comparten similitud temática entre sí. Esta similitud entre párrafos se establece cuando estos comparten los mismos patrones de palabras clave -o lemasentre sí. Cuando esto ocurre, se asume que hacen referencia a un mismo subtema. La idea de establecer clusters es individuar grupos de objetos que tengan dos características complementarias: que tengan la máxima homogeneidad interna, es decir, que los párrafos incluidos en el grupo sean lo más parecidos entre sí (en términos de patrones de lemas); y que tengan la máxima heterogeneidad externa, esto es, que dos párrafos sean lo suficientemente diferentes como para formar parte de dos grupos diferenciables entre sí. Esto es determinado automáticamente por el software.

En definitiva, así es como identificamos subtemas dentro del tema de la educación, y cómo se hablaba de ellos en las noticias, a través de las palabras clave que conformaron cada agrupamiento o clúster.

En Resumen, la lematización funcionó primero como apoyo para el procedimiento lexicométrico, que nos permitió contabilizar las unidades con significado autónomo presentes en el corpus, y luego, nos ayudó a elaborar el análisis temático que, al utilizar los párrafos como unidad de sentido a analizar, incorpora el significado dentro de un contexto (por ello se habla de contextos elementales). Solo incorporando este último factor podemos identificar las temáticas más relevantes -en lo que se denominan clusters-, sin transgredir el proceso de comunicación por el que cada una de las noticias fue construida. 
La relevancia de analizar esta radio en particular es que corresponde a una radioemisora que surge fuera de la región metropolitana, específicamente en la región del Biobío y de acuerdo con Ipsos (2014) se encuentra entre la más escuchadas en Chile en los últimos veinticinco años y a partir del primer semestre de 2014 se ha consolidado como la primera con mayor audiencia a nivel nacional.

\section{Resultados}

Como se ha indicado, el presente análisis se centra en aquellas noticias de orientación nacional cuyas características cuantitativas del corpus analizado son las siguientes: 1269 contextos elementales (párrafos); 49.980 palabras analizadas (ocurrencias), 7155 palabras distintas agrupadas en 4990 lemas.

Tabla 1. Principales lemas del corpus

\begin{tabular}{|c|c|c|c|}
\hline Lema & $\mathrm{N}$ & Lema & $\mathrm{N}$ \\
\hline Gobierno & 358 & Candidato presidencial & 62 \\
\hline Educación & 300 & Becas y créditos & 61 \\
\hline Derecha & 279 & Recurso & 61 \\
\hline Estudiante & 214 & Presidente & 59 \\
\hline Chile & 189 & Confech & 58 \\
\hline Congreso & 165 & Entregar & 52 \\
\hline Educación Superior & 153 & Institución & 52 \\
\hline Movilización & 117 & Acreditación & 51 \\
\hline Universidad & 116 & Región & 50 \\
\hline Bachelet & 96 & Establecimiento & 50 \\
\hline Concertación & 90 & Profesor & 50 \\
\hline Alumno & 87 & Público & 49 \\
\hline Propuesta & 84 & Movimiento estudiantil & 49 \\
\hline Educación de calidad & 83 & Proceso & 49 \\
\hline Asegurar & 78 & Terminar & 48 \\
\hline Realizar & 77 & Sistema & 47 \\
\hline Educación gratuita & 73 & Nuevo & 46 \\
\hline Colegio & 71 & Llegar & 46 \\
\hline Proyecto & 67 & Mayor & 46 \\
\hline Joven & 66 & Respecto & 45 \\
\hline Señalar & 66 & Lucro & 44 \\
\hline
\end{tabular}

Fuente: Elaboración propia a partir de las noticias publicadas en la página web de Radio Bío-Bío 
Como se evidencia en la tabla 1, los principales lemas que aparecen en el corpus nos indican una serie de temas y actores sociales que estuvieron presentes en los artículos de prensa durante el año analizado. De este modo tenemos los lemas Gobierno (358), Derecha (279), Educación de Calidad (83), Becas y créditos (61) y Presidente (59), que dentro de sus respectivos contextos elementales muestran la posición del gobierno de derecha de aquel entonces respecto a la educación, que consistió en centrar el problema en la "Calidad", moviendo el foco de las políticas hacia el aumento de recursos por medio de becas y créditos a los estudiantes. De igual modo, otra de las estrategias llevadas a cabo por el Gobierno fue intentar trasladar la discusión sobre la educación al Congreso (165); esto con la finalidad de descomprimir la presión estudiantil y los significativos niveles de apoyo que la ciudadanía otorgaba a las demandas del movimiento.

Otros de los actores representados en el corpus de noticias son los estudiantes (214), tanto a través del espacio de organización de la Confederación de Estudiantes de Chile CONFECH (58), como del más amplio movimiento estudiantil (49). También está representada la estrategia más relevante de estos actores, la movilización (117), que tuvo como principal finalidad asegurar la educación gratuita (83) y terminar (48) con la forma en que opera el sistema (47) educacional, denunciando y condenando públicamente el lucro (44) de algunas instituciones privadas de Educación Superior.

Finalmente, es posible reconocer otro conjunto de actores presentes en los lemas como son Bachelet (96), concertación (90), candidato presidencial (62), lo cual nos remite al contexto de campaña electoral que vivió el país durante el año 2013, y cómo esta se vinculaba a las demandas estudiantiles.

El total de artículos publicados durante el año 2013 fue de 430 noticias referidas al campo de la educación, lo que equivale a un promedio de 35,8 noticias por mes. La mayor ocurrencia de noticias la encontramos en el mes en abril (15,6\%) y la menor en el mes de febrero (2,6\%), lo cual es coherente por el período vacacional (3) que vive el país. Esto se puede ver representado en el gráfico 1 .

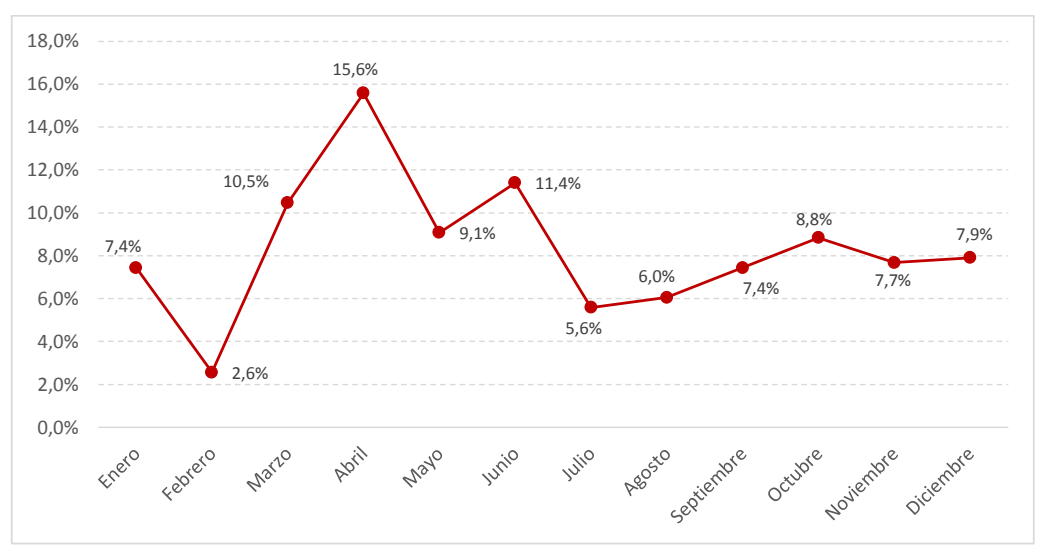

Grafico 1. Distribución porcentual de las noticias sobre educación, publicadas en la página web de Radio Bío-Bío durante el año 2013, por cada mes. 
En la tabla 2, se observa la orientación de las notas de prensa de acuerdo al nivel político administrativo territorial del que dan cuenta.

Tabla 2. Nivel de orientación de los artículos de prensa sobre educación, publicados en la página web de Radio Bío-Bío.

\begin{tabular}{|l|c|c|}
\hline \multicolumn{1}{|c|}{ Nivel } & $n$ & $\%$ \\
\hline Comunal & 95 & $22,1 \%$ \\
\hline Provincial & 14 & $3,3 \%$ \\
\hline Regional & 126 & $29,3 \%$ \\
\hline Nacional & 195 & $45,3 \%$ \\
\hline Total & 430 & $100,0 \%$ \\
\hline
\end{tabular}

Fuente: elaboración propia

Se evidencia el nivel nacional como el más relevante, seguido por el nivel regional, en donde el campo semántico de las noticias estuvo dado por los diferentes aspectos que llevaron a las secretarías regionales del Ministerio de Educación a manifestarse (inicio del año escolar, becas y explicaciones de la situación del sistema educacional); en cambio, en el nivel comunal los artículos de prensa estuvieron centrado en informar acerca de eventos, tales como robos en establecimientos educacionales, situaciones de violencia escolar y movilizaciones de los diferentes actores de la educación municipal. Finalmente, los tópicos informados en el nivel provincial son similares a lo encontrado en el nivel comunal (gráfico 2), como es posible observar en el plano factorial de cercanía entre ambos niveles.

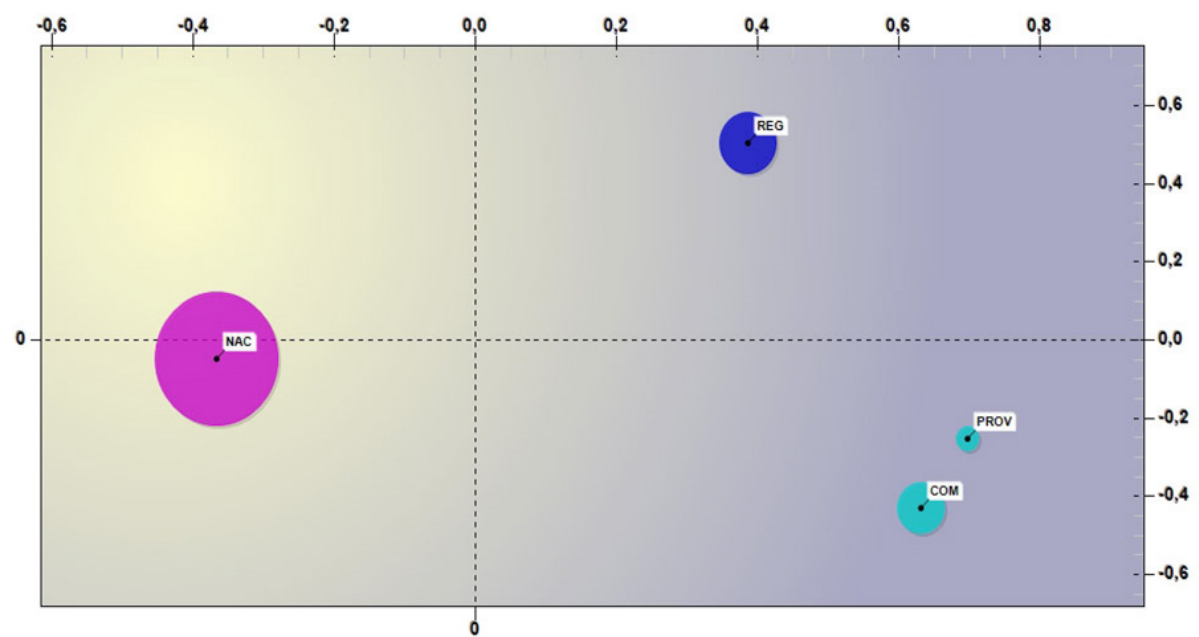

Gráfico 2. Plano factorial. Niveles político administrativo al cual se circunscriben las noticias (nacional, regional, provincial y comunal)

Fuente: Elaboración propia a partir de las noticias publicadas en la página web de Radio Bío-Bío 
Para identificar la temática presente en las noticias sobre la educación con orientación nacional, se utilizó un análisis temático de contextos elementales. Este procedimiento hace una representación de los contenidos del corpus mediante clusters temáticos de modo que: a) Cada cluster está constituido por un conjunto de contextos elementales (en nuestro caso párrafos) caracterizados por los mismos patrones de palabras clave (lemas); b) Cada cluster puede ser descrito por las unidades lexicales (lemas en nuestro caso) que más caracterizan los contextos elementales de los cuales se compone.

De este modo, es posible identificar el porcentaje de unidades de contexto (párrafos) que pertenecen a cada cluster: Así, el Cluster 1, "Gobierno", representa el 23,3 \%, el Cluster 2 "Movilización", el 46,6 \% y el Cluster 3 "Universidad", el 30,1%.

En el gráfico 3 se visualizan en el plano cartesiano (eje X, Y) las correspondencias entre las palabras y los cluster (temas emergentes). La posición de cada palabra y cluster en el mapa, se encuentra determinada por los índices de asociación de cada uno de ellos, lo que significa que a mayor cercanía entre puntos (palabras y cluster) mayor relación existe entre ellos (grupos de redes de palabras y párrafos tienden a ser comunes; por lo tanto, hay mayor relación temática), mientras que a menor cercanía (o mayor lejanía) entre puntos, menor es la relación temática entre ellos, es decir, cada uno posee párrafos y palabras clave que tienen a diferir y alejarse respecto a la otra.

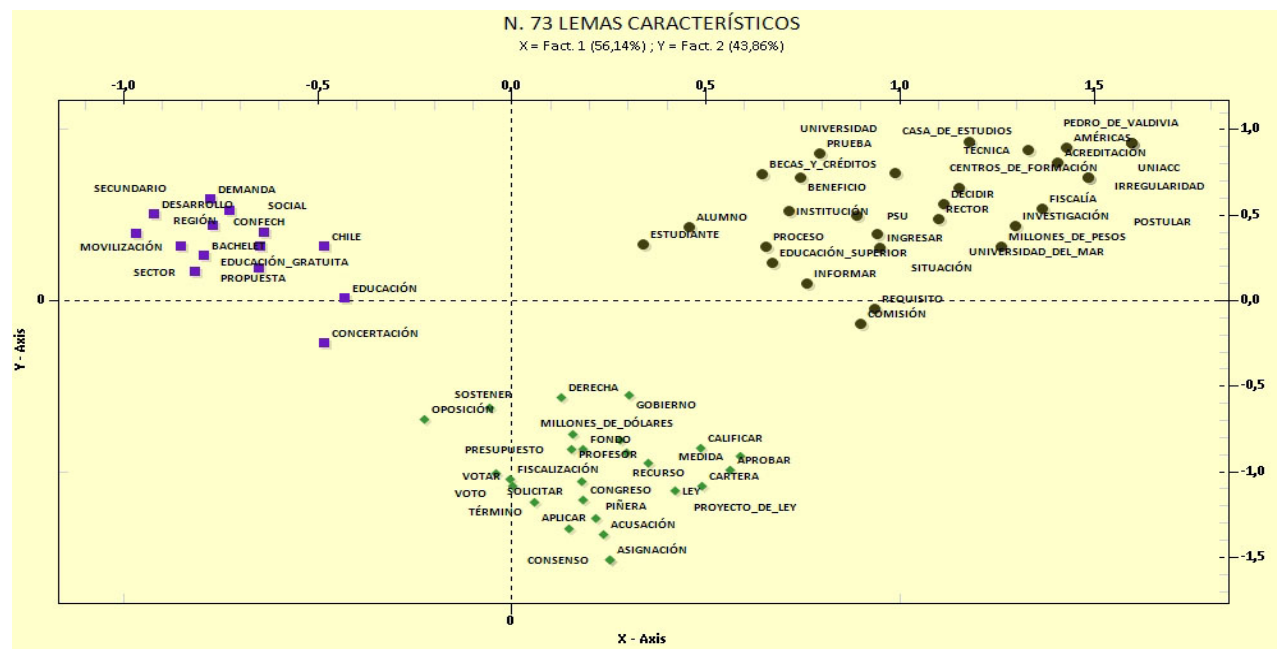

Gráfico 3. Distribución de Lemas organizados en tres cluster temáticos.

Fuente: Elaboración propia a partir de las noticias publicadas en la página web de Radio Bío-Bío.

Cluster 1: Gobierno. Se ha identificado este cluster con el rótulo de Gobierno, en tanto agrupa aquellos lemas vinculados con dicha familia semántica, en donde se representa la reacción del sistema político, específicamente del Gobierno, en oposición a las reformas postuladas por el movimiento estudiantil.

En la representación de este tema, podemos observar que los lemas que caracterizan el grupo hacen referencia a la respuesta del Gobierno, orientada a generar reformas tras 
acuerdos políticos en el Congreso, que permitiesen aprobar una legislación centrada en mejoras a la calidad del sistema educativo, además de buscar aprobar el aumento de recursos del presupuesto destinado a educación, sin considerar cambios estructurales. Las respuestas del Gobierno a la crisis del sistema educacional partían del supuesto de que el problema surge de las expectativas de los sujetos, y que este se soluciona al mejorar la calidad de la educación, no a través de un cambio en la estructura del sistema educacional. Esto lo podemos ejemplificar a través de los siguientes extractos de noticias, incluidos en este cluster:

La reforma más importante que estamos llevando a cabo como sociedad hoy día en Chile es la reforma a la educación. Y esta es una reforma en la cual simplemente no podemos fallar y es por esa razón que el Gobierno ha comprometido los recursos necesarios para mejorar la calidad de la educación en Chile, pero esto no es solamente un problema de recursos, esto requiere un fuerte compromiso (Radio Bío-Bío, 10/04/2013).

La ministra de Educación, Carolina Schmidt, no quiso entrar en polémica con los estudiantes y resaltó la importancia de aumentar el número de becas para las familias del 60 por ciento más vulnerable, única posibilidad para que muchos jóvenes con talento puedan ingresar a la educación superior... Según la secretaria de Estado, "el presupuesto 2014 está concentrado, de manera muy importante, en Educación, que concentra 21 \% del gasto público total del país, dejando a Chile como uno de los países con mayor proporción de inversión pública en Educación del mundo", afirmó, agregando que "la prioridad para el Gobierno del Presidente Piñera ha sido la inversión en Educación. Uno de cada cinco pesos del Presupuesto total del sector público va a Educación" (Radio Bío-Bío, 03/10/2013).

Cluster 2: Movilización. Aquí se encuentran los lemas asociados a los estudiantes secundarios y universitarios, cuya representación está dada por el posicionamiento de sus demandas a través de la formulación de propuestas, acompañada de la movilización. Como antecedente indicamos que las demandas levantadas en 2011, consistieron principalmente en lograr la "educación gratuita, fin al lucro, democratización, fin al endeudamiento y el autofinanciamiento y acceso equitativo" (CONFECH, 2011, p.1). Ejemplificamos esta representación a través de los siguientes extractos:

Este jueves se realizó la primera marcha convocada por organizaciones estudiantiles, que fue encabezada por el Movimiento de Estudiantes de Educación Superior Privada (Mesup), y que contó además con el apoyo de los secundarios, la Aces, la agrupación de estafados por el crédito Corfo, y estudiantes afectados por el escándalo de la Universidad del Mar... (Radio Bío-Bío, 28/03/2013).

La Confederación de Estudiantes de Chile (Confech) llamó a un nuevo paro nacional por la educación para el próximo 26 de junio, movilización que se suma a la marcha convocada para el día 13 del mismo mes. Esto, por las nulas respuestas que, a su juicio, el Ejecutivo ha dado a las demandas del movimiento estudiantil, lo que los obligaría a reimpulsar las protestas, explicó el presidente de la Federación de Estudiantes de la Universidad Alberto Hurtado, Pablo Flores (Radio Bío-Bío, 02/06/2013). 
En este cluster se encuentran presentes también "Bachelet" y "Concertación", lo cual se explica por la campaña electoral del año 2013, puesto que la actual mandataria en ese momento era candidata presidencial. En ese entonces, su coalición asume en su proyecto político, al menos en lo discursivo, algunas de las demandas del movimiento estudiantil, tales como la gratuidad en la Educación Superior y el fortalecimiento de la educación pública.

Cluster 3: Universidad. Este cluster reúne al conjunto de lemas que dan cuenta de la representación del sistema de Educación Superior. Aquí podemos observar el estrecho vínculo entre la aparición de universidades privadas -Universidad del Mar, de las Américas, Pedro de Valdivia, UNIACC-, y los lemas "Irregularidad", "Fiscalía", "Investigación" y "Millones de pesos", de lo que desprendemos que el medio de comunicación representó de manera relevante el problema del lucro en el sistema de Educación Superior. Como forma de contextualizar indicamos que durante el año 2013, tras el escándalo de la venta de acreditaciones a universidades privadas y cuyo caso emblemático fue la Universidad del Mar, la preocupación del Ministerio de Educación estuvo dada por la idea de no tolerar las irregularidades del sistema, y por la situación de los estudiantes de dichas casas de estudios que, tras su cierre, debieron ser ubicados en otras universidades. De esto dan cuenta los siguientes extractos:

El presidente de la Comisión Nacional de Acreditación, Matko Koljatic, adelantó en exclusiva a Radio Bío Bío, el inicio de una investigación a una de las agencias acreditadoras por irregularidades en sus procedimientos [.. .] Sin embargo, la situación del plantel sólo ha llevado a ampliar el espectro de las universidades privadas investigadas, salpicando incluso a la Comisión Nacional de Acreditación, debido a las irregularidades cometidas por su ex presidente, Luis Eugenio Díaz [...] A esto hay sumar la investigación administrativa que iniciará en los próximos días el Ministerio de Educación a la Universidad SEK, luego de la formalización por soborno del ex rector José Schroeder (Radio Bío-Bío, 14/03/2013).

El Ministerio de Educación detectó irregularidades en la investigación administrativa en contra de la Uniacc y la Universidad Pedro de Valdivia, y remitirá los antecedentes al Ministerio Público [...] la CNA envió los antecedentes a la cartera de Educación para que se evalúe si se amerita iniciar una investigación administrativa al plantel, como lo detalló Alberto Vásquez (Radio Bío-Bío, 28/10/2013).

Además, podemos observar que aparecen lemas asociados al ingreso al sistema de Educación Superior, como "PSU", "beneficio", "becas y créditos", que representan el proceso de ingreso en relación a posibilidades de financiamiento para los estudiantes.

\section{Discusión y conclusiones}

Por medio de procedimientos automáticos hemos identificado la representación social que tiene sobre la educación el que es reconocido por Ipsos (2014) como uno de los principales medios de comunicación de masas de nivel nacional: la Radio Bío-Bío. 
Los principales hallazgos del estudio nos indican que, a partir del procedimiento de lematización es posible identificar los tópicos: Educación Superior, Movilización, Educación de Calidad y Educación Gratuita, y los principales actores sociales involucrados: Gobierno, Derecha, Congreso, Estudiantes, Bachelet y Concertación. A partir de este procedimiento fue posible detectar redes temáticas de sentido, en donde, se evidencia la relación entre gobierno, derecha, educación de calidad Congreso y becas-créditos, que dan cuenta de la estrategia del Gobierno pasado para enfrentar el conflicto. En oposición a esta red de sentido, desde los estudiantes, CONFECH, movimiento estudiantil, movilización, educación gratuita, terminar, sistema y lucro, lemas que representan las demandas por la cual se articuló de manera clara el movimiento por la educación en 2011.

Por medio de los análisis temáticos de contextos elementales (lemas dentro de su contexto) fue posible identificar tres cluster (agrupamientos) de noticias: movilización, universidad y gobierno. Cada uno de éstos correspondería a la representación que realizó este medio de comunicación durante el año 2013, respecto del tema de la educación, permitiéndonos conocer cómo la radio organizó temáticas y actores, con sus respectivas posiciones y estrategias.

Si entendemos las representaciones sociales, como lo sostiene Martín Serrano (1977), como modelos construidos que sirven para orientar la acción y la valoración de los sujetos, describir las representaciones sociales sobre la educación de un medio masivo de comunicación, tan relevante como la Radio Bío-Bío nos permite acceder a la forma en que ese medio de comunicación construyó un modelo interpretativo sobre la realidad del conflicto por la educación durante el 2013, pero también nos entrega elementos para aproximarnos a la forma en que sus lectores pudieron haber orientado sus valoraciones y acciones respecto del mismo conflicto, teniendo en claro que esto último supera las pretensiones del presente trabajo.

El presente estudio dio cuenta del tratamiento que se hizo sobre la educación en este medio de comunicación, durante el año 2013, bajo un contexto de gobierno de derecha y campaña presidencial. Cabe indicar que dichos resultados son el inicio de un estudio longitudinal y a partir del conjunto de noticias que se vienen presentando durante el presente año, lo que nos permitirá en el futuro poder comparar tal representación bajo un contexto diferente, posición política del gobierno, institucionalización de dirigentes estudiantiles y reformas en distinto ámbito que son, vale recordar, un producto del conflicto.

\section{NOTAS}

(1) La LOCE fue la Ley Orgánica Constitucional de Enseñanza, promulgada por la Junta Militar en marzo de 1990, a solo días de traspasar el mando al gobierno democráticamente electo de Patricio Aylwin. La institucionalidad educacional que fijó dicha ley no fue significativamente modificada por los gobiernos de la Concertación, coalición de centro-izquierda que presidió el Poder Ejecutivo en los períodos de 1991-1994, 1994-2000, 2000-2006, y 2006-2010.

(2) CONFECH es la Confederación de Estudiantes de Chile, organización que reúne a las federaciones de estudiantes de las universidades pertenecientes al Consejo de Rectores, instancia que aglomera a universidades estatales, y aquellas consideradas tradicionalmente "públicas". Las organizaciones de 
estudiantes secundarios más relevantes de ese momento fueron la ACES, Asamblea Coordinadora de Estudiantes Secundarios, y la CONES, Coordinadora Nacional de Estudiantes Secundarios.

(3) En Chile, el período vacacional estudiantil oscila entre mediados de diciembre y finales de febrero.

\section{Referencias bibliográficas}

Adimark (2011). Evaluación de Gobierno. Agosto 2011. Recuperado el 20 de junio de 2016. Disponible en http://www.adimark.cl/es/estudios/archivo.asp?id=130

CONFECH (2011). "Petitorio Confech". Actualizado al 26 de junio. Disponible en: http://fech.cl/ blog/201 1/07/28/compilado-documentos-de-interes-movilizaciones-2011-2/petitorio-confech/

Orellana, C. (Enero de 2014). El conflicto por la educación en Chile. Conferencia llevada a cabo en el Instituto de Prospectiva, Innovación, y Gestión del Conocimiento, Facultad de Ciencias Administrativas de la Universidad del Valle, Cali, Colombia.

Jodelet, D. (1984). La representación social: fenómenos, conceptos y teoría. En S. Moscovici (Comp.), Psicología Social. Barcelona: Paidós.

IPSOS (2014). Radio al Aire. Disponible en: http://www.ipsos.cl/ipsosradioalaire/index.htm

Martín Serrano, M. (1977). La mediación social. Madrid: Akal.

Martinic, S. (2006). "El estudio de las representaciones y El Análisis Estructural del Discurso". En M. Canales (Ed.), Metodologías de investigación social. Introducción a los oficios (pp.299-319). Santiago de Chile: LOM. 
